\title{
ENQUÊTE LITTÉRAIRE, EN QUÊTE DE SOI : LE COMPLEXE DE LA SORCIÈRE D'ISABELLE SORENTE
}

Dans Le complexe de la sorcière (2020), Isabelle Sorente raconte comment l'image de la sorcière fait irruption dans sa vie et dans son écriture, et l'amène à interroger l'impact psychique des chasses aux sorcières, ainsi que sa transmission tacite aux générations postérieures. Si la littérature contemporaine « revendique volontiers l'enquête comme démarche d'écriture" (Laurent Demanze, Un nouvel âge de l'enquête, 2019), Le complexe de la sorcière illustre cette tendance en alliant enquête historique, exploration de la mémoire familiale, auto-analyse et introspection. En étudiant le dernier roman d'Isabelle Sorente, nous nous intéresserons à ce mode particulier de représentation littéraire de la vie intérieure, où l'écriture conçue comme enquête devient le véhicule d'une transformation intérieure. En poursuivant une analyse parallèle de la prise de conscience de l'auteure et des transformations génériques que subit le récit, nous verrons que l'enquête transforme d'abord le roman en essai, et le rapproche de la notion de littérature appliquée à la psychanalyse (P. Bayard). Nous verrons ensuite que la remémoration du passé familial et personnel, au lieu d'être l'aboutissement de la narration, ouvre le récit de soi aux récits des autres. Finalement, les réflexions de P. Ricœur nous permettront d'articuler la relation entre littérature documentaire et construction du sujet.

Mots clés : Isabelle Sorente, Le complexe de la sorcière, enquête, femmes, hybridité générique, littérature française du $21^{\mathrm{e}}$ siècle, sorcières, vie intérieure.

\section{INTRODUCTION}

C'est à l'heure d'une revalorisation évidente de la sorcière au sein de la culture populaire ${ }^{1}$, qu'Isabelle Sorente publie son dernier roman², intitulé $L e$

\footnotetext{
*nadja.djuric@fil.bg.ac.rs

1 Pour ce qui est de la revalorisation de la sorcière dans la culture populaire, en témoigne de manière exemplaire le numéro hors-série du magazine Le Point, intitulé Les Sorcières: Histoire d'une renaissance, paru en novembre-décembre 2019, et
} 
complexe de la sorcière (2020). Le pouvoir magique de la sorcière, son savoir qui s'oppose au savoir institutionnel, son statut marginal, sa proximité avec la nature, font d'elle une figure emblématique de certaines tendances contemporaines, culturelles ou politiques (tendances féministes surtout, mais aussi écologiques ou anticapitalistes).

Le regain de popularité que connaissent les sorcières dans les dernières années ne saurait donc surprendre, d'autant plus que cette revalorisation d'une figure autrefois proscrite possède une histoire. Dans La sorcière, l'essai incontournable de Jules Michelet dont la première édition date de 1862, l'historien ne cache pas sa compassion pour la femme chassée, "honnie, tiraillée, lapidée, assise sur les charbons ardents » (Michelet, 1878 : V) et se propose justement d'en donner une image positive. Environ un siècle plus tard, au début de son texte "Quelques observations sur la sorcellerie européenne », l'historien de religion Mircea Eliade se dit impressionné par la popularité de la sorcellerie dans la culture occidentale moderne (Eliade, 1978 : 93). Avec Le complexe de la sorcière, Isabelle Sorente participerait alors à une nouvelle étape de cette évolution, conditionnée par les préoccupations de la société contemporaine.

reflétant de manière claire le regard que pose la société contemporaine sur la sorcière. L'histoire des sorcières y est retracée en quatre segments: «Le temps des magiciennes », avec des articles sur les sorcières romaines, ou encore sur Médée ou Circé ; «La grande chasse », où l'on aborde les chasses aux sorcières qui ont eu lieu en Europe du XVe au XVIII e siècles; "Le retournement», où l'on s'intéresse au changement progressif du statut de la sorcière au XVIII e et XIXe siècles; et finalement "Le retour en gloire", consacré aux facettes nombreuses que connaît le phénomène des sorcières de nos jours, de la rupture avec le patriarcat et la recherche de spiritualité, à la sorcellerie comme profession. L'état actuel est constaté sans réserve : la sorcière est devenue une nouvelle icône du féminisme. Dans cette même veine, on pourrait évoquer un autre ouvrage, Sorcières: la puissance invaincue des femmes de Mona Chollet (2018), décidemment féministe lui aussi, où l'auteure, journaliste de profession, met en relation l'histoire de la sorcière avec les questions de l'indépendance féminine, de la stérilité voulue, problématise la vieillesse féminine, et s'interroge sur les problèmes que pose le rapport à la nature dans la société contemporaine.

2 Isabele Sorente est auteure de huit romans : $L$ (2001), La prière de septembre (2002), Le coeur de l'ogre (2003), Panique (2006), Transformations d'une femme (2008), 180 jours (2013), La faille (2015), Le complexe de la sorcière (2020). Elle a publié également des essais : La femme qui rit: le marché noir de la réalité (2007), Addiction générale (2011), État sauvage (2012). Ses ouvrages n'ont pas encore été traduits en serbe. 
La thématique de l'ouvrage est clairement féministe : le fil directeur du roman se tisse autour des questions de la souffrance des femmes, de la quête d'indépendance féminine (spirituelle, avant tout), ainsi que de la recherche de l'authenticité dans la vie intérieure des femmes. Ce sont des thèmes auxquels Isabelle Sorente s'est déjà intéressée; son avant-dernier roman, La faille, racontait justement l'histoire d'une jeune femme se trouvant sous l'emprise destructrice de son mari, pervers narcissique, et problématisait la susceptibilité de l'héroïne à ce type d'influence (Sorente, 2015). Dans Le complexe de la sorcière, l'auteure traite donc de nouveau un de ses sujets de prédilection. Le fait de se servir de la figure de la sorcière pour aborder une problématique féministe n'est pas novateur en soi, mais la manière dont elle choisit d'actualiser cette figure apporte une perspective innovante, authentique et pertinente, à la réinvention de la sorcière en tant qu'héroïne féministe.

Nous présenterons d'abord le complexe élaboré par Isabelle Sorente, mais il ne sera pas le sujet proprement dit de notre étude. Nous nous intéresserons davantage à la manière dont l'auteure, s'étant approprié la figure de la sorcière, plie son écriture aux besoins de sa vie intérieure. Car l'écriture est, pour Isabelle Sorente, non seulement le moyen de représenter l'intériorité et d'en éclaircir les zones d'ombre, mais aussi le moyen d'arriver à une transformation intérieure. Et si la littérature contemporaine «revendique volontiers l'enquête comme démarche d'écriture » (Demanze, 2019 : 14), dans sa recherche d'une vérité intérieure Isabelle Sorente se sert justement du procédé de l'enquête. Le complexe de la sorcière partage la même orientation herméneutique que l'on retrouve dans la «littérature du réel » dont Laurent Demanze étudie les dispositifs, l'abordant sous ses formes variées à partir de l'émergence du récit herméneutique au XIXe siècle (Demanze, 2019 : 39), jusqu'aux modalités contemporaines de l'investigation littéraire. Cependant, le texte d'Isabelle Sorente présente une spécificité importante: il est question d'appliquer le principe de l'enquête sur sa propre vie intérieure, et c'est cette manière d'allier le principe de l'enquête avec l'écriture de l'intériorité, que nous nous proposons d'étudier dans les pages qui suivent. Après avoir expliqué la signification du complexe éponyme, nous montrerons comment dans Le complexe de la sorcière un projet de roman à peine ébauché se retrouve soumis à plusieurs transformations, oscillant entre genres littéraires ou pratiques d'écriture, puisqu'il s'agit à la fois d'un roman, d'un essai et d'un récit de soi. Nous verrons également que ce texte hybride instaure une dynamique 
créatrice entre le passé évoqué et le présent de l'écriture, investissant ainsi l'écriture d'un pouvoir de transformation intérieure.

\section{QU'EST-CE QUE LE COMPLEXE DE LA SORCIÈRE?}

Pour définir un complexe, les interlocuteurs de l'auteure ont recours à l'image de la constellation, dont on peut voir certaines étoiles alors que d'autres restent invisibles (Sorente, 2020 : 76). Cette comparaison vient en fait de C. G. Jung : pour lui, la constellation «est une opération automatique, spontanée, involontaire, dont personne ne peut se défendre », alors que les « contenus constellés répondent à certains complexes » (Jung, 1987 : 184, empl. 3497), le complexe affectif étant défini comme « l'image émotionnelle et vivace d'une situation psychique arrêtée, image incompatible, en outre, avec l'attitude et l'atmosphère conscientes habituelles » (Jung, 1987 : 187, empl. 3541). Pour Isabelle Sorente, la sorcière est justement une constellation, possédant un côté sombre et un côté lumineux (Sorente, 2020 : 77).

Le propos central de l'ouvrage pourrait tenir en peu de mots : Isabelle Sorente raconte comment l'image de la sorcière fait irruption dans sa vie et dans son écriture, et l'amène à interroger l'impact psychique des chasses aux sorcières, ainsi que sa transmission tacite aux générations postérieures. Selon l'auteure, la violence faite aux femmes durant les chasses aux sorcières a laissé une empreinte qui se transmet d'une génération des femmes à l'autre, à leur insu même, et qui ne cesse pas de faire des ravages dans leur vie intérieure.

Le complexe de la sorcière serait ainsi le produit d'une expérience historique qui s'est transformée à travers les siècles : de l'action directe et concrète de l'inquisiteur, homme en chair et en os, qui s'est donné pour tâche de briser l'esprit de la femme accusée, de s'approprier sa vérité psychique pour l'anéantir, on passe à l'instauration d'une culture basée sur la peur et la négation de soi, puisqu'elles deviennent nécessaires afin d'assurer la survie de la femme, pour arriver finalement, par le biais de l'intériorisation et de la transmission intergénérationnelle, au complexe de la sorcière, qu'il faudrait comprendre comme une constellation psychique agissant de manière subreptice pour fausser le rapport des femmes à elles-mêmes, ce qui peut aller de la dépréciation socialement valorisée (« Ce discret soupçon de soi-même, si adorable n'est-ce pas, parce que c'est adorable une femme qui doute, alors qu'une femme qui ne doute pas... », Sorente, 2020 : 68), jusqu'à la haine de soi et à la peur d'être anéantie ("'pitié ne me tuez pas', c'est le refrain de cette 
peur excessive »Sorente, 2020 : 228, 247). Le rôle de l'inquisiteur peut toujours être joué par un homme concret, ou par les diverses institutions de pouvoir, mais l'originalité de l'approche d'Isabelle Sorente réside justement dans ce lien qu'elle établit entre la relation Sorcière-Inquisiteur, et la vie intérieure des générations de femmes. L'accusée d'hier ou sa descendante d'aujourd'hui se disent toutes les deux : «Il y a dans ma conscience un principe trompeur. Je ne peux croire en moi. Je ne peux croire qu'en vous. Ma conscience est incompétente. Je renonce à ma conscience » (Sorente, 2020 : 6768). Dans Le complexe de la sorcière, la figure de la sorcière apparaît donc dans deux dimensions temporelles : évoquant l'historicité du phénomène, l'auteure tient à informer le lecteur de manière détaillée sur les chasses aux sorcières, chiffres à l'appui ; mais elle procède aussi à son actualisation, dans la mesure où il s'agit de dialoguer avec la part de la sorcière en soi ${ }^{3}$.

\section{DU ROMAN À L'ESSAI...}

Par-delà l'actualité de la thématique, la forme même du livre d'Isabelle Sorente illustre de manière nette une des tendances importantes de la littérature contemporaine : il est question de cette hybridité générique qui devient de plus en plus caractéristique de la production littéraire actuelle, puisque les frontières entre les genres sont tout à fait poreuses et se brouillent facilement. D'ailleurs, sur la $4^{\mathrm{e}}$ de couverture, on peut lire que Le complexe de la sorcière est "le récit d'une enquête", enquête historique de plus, mais que cette reconstitution de l'histoire des grandes chasses est en même temps « un roman bouleversant sur l'adolescence, la mémoire familiale ». Justement, l'ouvrage alterne entre l'enquête historique, l'exploration de la mémoire familiale, l'auto-analyse et l'introspection, d'une manière qu'il nous faudrait étudier de plus près.

Dans le premier chapitre, intitulé «Apparition », il est question surtout du brouillage de la frontière entre la fiction et la littérature documentaire. Le roman s'ouvre justement sur une apparition : est évoquée, au tout début, « une

${ }^{3}$ De plus, on constate l'intention de réinventer le sabbat en tant que réunion des femmes : en fait, parmi les personnages secondaires, on compte surtout les amies de l'écrivaine, et c'est par des échanges qu'elle peut avoir grâce au fait d'appartenir à un réseau de femmes, qu'elle arrive à formuler et à approfondir ses idées. 
scène terrible, une scène d'interrogatoire » (Sorente, 2020 : 11), où un homme se tient derrière une femme, et lui demande d'avouer qu'elle est une sorcière. Seulement, ce n'est pas un début du roman, historique ou autre, ou alors c'est le début d'un roman qui ne sera pas écrit, puisque l'écrivaine, même si elle est hantée par cette image, n'arrive pas à construire une histoire autour d'elle. Le récit que nous lisons se présente dans un premier temps comme le récit de la préparation à l'écriture d'un texte de fiction - un texte qui ne verra pas jour. On peut constater alors un premier détournement de l'écriture par lequel on abandonne l'ambition romanesque pour écrire, au fond, un texte documentaire : c'est comme si le paratexte du roman que l'auteure envisageait d'écrire se mettait à la place du texte lui-même, comme si le contenu de la préface ou du journal d'écriture prenaient la place du texte romanesque luimême.

$\mathrm{Au}$ lieu de nous raconter l'histoire de la sorcière qui lui est apparue, au lieu de créer une histoire fictionnelle en s'appuyant sur des textes documentaires, l'auteure revient donc vers une histoire qui a réellement eu lieu, celle des grandes chasses. Cette partie du texte présente un double enjeu : elle se veut explicative, avec une intention visible d'éduquer et d'informer le lecteur, mais elle raconte en même temps la découverte faite par l'auteure ellemême. Non seulement elle découvre la portée véritable des chasses, mais elle se rend également compte d'un fait terrifiant, qu'elle avait jusque-là méconnu : les chasses aux sorcières ne sont pas l'héritage de l'époque médiévale; au contraire, elles ont marqué le début même de l'époque moderne. La découverte de l'Histoire déplace définitivement le récit du côté de la littérature documentaire, et le rapproche même de l'essai. Isabelle Sorente présente et commente les livres de son corpus, ouvrages censés servir au travail préparatoire de l'écriture; ouvrages historiques, certes, mais également des ouvrages sur la psychogénéalogie, ainsi que des ouvrages de fiction dans lesquels elle retrouve la thématique qui l'intéresse ${ }^{4}$. En plus de s'approprier son propre paratexte, le roman intègre sa bibliographie commentée et énumère de manière précise les influences en matière de la représentation de la sorcière; d'une certaine manière, on pourrait considérer qu'il a même devancé les textes critiques dont il pourrait susciter l'écriture.

${ }^{4}$ Il est question, par exemple, de livres de Doris Lessing, de Christa Wolf ou d'Ingeborg Bachmann. 
Puisant dans des recherches déjà menées, le livre s'ouvre ainsi à des domaines divers de connaissance, mais il se constitue aussi lui-même comme le lieu de l'élaboration d'une connaissance. Le titre fait référence à la psychanalyse, en insistant sur la notion de complexe, puisque l'ambition de l'auteure dépasse largement la reconstitution historique: Isabelle Sorente souhaite réfléchir aux répercussions de l'événement historique sur la vie psychique des femmes, de l'époque des chasses jusqu'à nos jours. Le titre choisi souligne l'importance de la psychanalyse, qui est d'emblée posée comme modèle de travail interprétatif, permettant de se connaître et de donner un sens à son expérience personnelle. Mais ce modèle suscite également une attitude quelque peu ambivalente : le nouveau complexe proposé par Isabelle Sorente aurait pu, en théorie, chercher sa place ailleurs que dans la littérature ; il vacille entre le sérieux de la psychanalyse dont il s'inspire, et les simulations de la littérature qui peuvent aider le sujet à se construire, sans pour autant prétendre à un statut scientifique. En tant qu'invention littéraire, il est à la fois autorisé et exclus par la psychanalyse.

Pour comprendre l'enjeu d'une écriture qui se situe d'une manière si équivoque entre la littérature et la psychanalyse, il serait utile de se référer au propos développé par Pierre Bayard dans Peut-on appliquer la littérature à la psychanalyse ? Bayard distingue deux démarches: alors que la psychanalyse appliquée à la littérature cherche dans les textes littéraires une confirmation de ses théories, la littérature appliquée (à la psychanalyse) "procèderait de manière inverse et tenterait de ne pas projeter sur les textes littéraires une théorie extérieure, mais, au contraire, de produire de la théorie à partir de ces textes » (Bayard, 2012, empl. 536). Ainsi, la première démarche est basée sur la notion du sens inconscient, la seconde sur celle de la pensée virtuelle, puisqu'il faut se demander «de quelle pensée originale elle [l'œuvre] est dépositaire» (Bayard, 2012, empl. 549). De plus, selon Bayard, l'un des modèles du psychisme différents de ceux élaborés par Freud serait à chercher dans certains textes de Romain Gary. C'est la ventriloquie des personnages de Gary qui intéresse Bayard, et l'amène à évoquer une topique de la possession : Gary confère " une véritable existence séparée à une partie du psychisme qui serait, à le suivre, peuplée de créatures humaines éloignées ou disparues », dont la « présence n'a rien d'inconscient, puisque c'est précisément ce dialogue plus ou moins volontaire avec les anciens qui nous constitue comme sujets » (Bayard, 2021, empl. 1835). Lorsqu'elle établit le complexe de la sorcière et, qui plus est, instaure un dialogue intérieur avec elle, Isabelle Sorente procède 
de manière analogue, mais avec une différence décisive : sa pensée virtuelle se développe dans le cadre même de la psychanalyse. Le passage du roman à l'essai correspondrait alors à une situation du clivage entre la psychanalyse appliquée et la littérature appliquée.

\section{... ET DE L'ESSAI À L'ÉCRITURE DE SOI}

Il s'avère pourtant que ce passage du roman à l'essai n'est que le premier mouvement dans la transformation générique du texte. Dans le premier chapitre le propos de l'auteure est remarquablement clair et son intention évidente. Si la lecture de l'ouvrage, lorsqu'on arrive à la fin de ce chapitre, provoque une certaine incertitude, celle-ci porte sur la direction que le « roman » pourrait prendre par la suite : le texte va-t-il revenir vers un modèle d'écriture romanesque plus traditionnelle, pour raconter bel et bien une histoire, ou alors renoncera-t-il définitivement à l'allure de la fiction pour se transformer en ouvrage théorique? Et si une histoire serait finalement à raconter, que pourrait-elle prendre pour sujet : le destin d'une femme accusée jadis de sorcellerie, ou celui d'une femme qui subit, sans le savoir, les conséquences néfastes du travail sournois du complexe de la sorcière?

C'est à ce moment qu'Isabelle Sorente surprend le lecteur par un autre détournement générique, en introduisant une thématique qui, bien que dans la continuité directe avec le sujet précédemment évoqué, n'est pas vraiment possible à anticiper. Cette fois-ci, le récit porte sur sa propre expérience, et donne à la notion des "Chasses » (c'est d'ailleurs le titre du second chapitre) une portée plus large et plus universelle. L'auteure se tourne vers ses années d'adolescence pour se souvenir d'une période où elle a été littéralement chassée; dire qu'elle a été pendant des années «le souffre-douleur de [s]a classe » (Sorente, 2020 : 117), comme le constate l'auteure elle-même, ne suffit pas pour rendre compte de l'harcèlement qu'elle a dû subir. Dans ces pages, difficiles à lire en raison de la nature même de l'expérience évoquée (d'autant plus qu'on ne s'attend pas à ce que le récit prenne cette tournure), le travail de remémoration fait ressurgir un passé que l'auteure aurait visiblement préféré laisser dans l'oubli, mais que la sorcière - incarnation de la recherche d'une vérité psychique - lui impose de revivre.

Ce passé est contextualisé et expliqué à l'aide de la perspective nouvellement acquise : la persécution de l'adolescente est interprétée comme une des manifestations de la chasse, ce qui permet à l'auteure de généraliser la 
notion du complexe de la sorcière, d'en reconnaître le paradigme par-delà les situations où des femmes sont opprimées par des hommes, que ceux-ci représentent ou non un pouvoir institutionnel. En même temps, pour revenir à la question de l'hybridité générique, l'enquête historique se transforme en enquête de son propre passé par le biais du complexe, qui permet de les associer à travers la perspective intergénérationnelle. De quel type de récit serait-il question maintenant ? Comment distinguer la part du documentaire et la part du fictionnel dans ce deuxième chapitre ? L'écriture s'apparente-t-elle davantage à l'autobiographie, à l'autofiction, au témoignage ? La frontière entre le vécu et sa transposition littéraire reste impossible à tracer pour le lecteur, bien qu'il soit évident que la volonté de l'écrivaine est de témoigner de sa propre expérience.

Le témoignage de l'écrivaine s'inscrit dans le cadre d'une " analyse avec la sorcière », une auto-analyse pour laquelle l'auteure doit se préparer et se munir d'outils théoriques et pratiques elle-même, puisque le complexe qui la travaille n'est pas officiellement reconnu. La véritable intrigue du roman concerne donc sa vie intérieure, sans aucun support événementiel - c'est une prise de conscience qu'on raconte - et la résolution du conflit relève également d'une expérience intérieure. Le troisième chapitre, "Pardon », rend justement le pardon possible par l'intermédiaire de l'aveu du père de l'auteure, coupable lui-même d'avoir infligé de telles souffrances durant sa jeunesse, mais il ne s'agit pourtant pas d'un dénouement. Le tout dernier chapitre, "Sabbat», raconte la réunion et les histoires partagées entre l'auteure et ses amies, qui reviennent sur la manière dont la sorcière est apparue dans leur vie, et dont elles ont "bifurqué » pour arriver à reconnaître leur vérité psychique et spirituelle. Aucune révélation merveilleuse, pourtant, aucune transformation prodigieuse et foudroyante: il est question de quelques expériences individuelles, concrètes et crédibles, et d'une série de réflexions inspirées par les diverses manières dont on peut regagner sa vérité de femme.

Cette vérité ne saurait être figée ni définitive, et l'éparpillement du récit dans sa partie finale traduit la volonté d'éviter une clôture narrative conçue comme dénouement. Ce choix n'a rien de surprenant, car l'auteure n'a jamais cessé de déplacer l'enjeu et l'intérêt de son récit : il a fallu d'abord renoncer à raconter l'histoire de la sorcière, puis renoncer à rédiger l'Histoire des sorcières, et se refuser ensuite de poursuivre sa propre histoire jusqu'à la fin du récit. Ces renoncements et ces déplacements sont autant de signes d'une méfiance envers la maîtrise du récit, héritée d'ailleurs des romanciers majeurs 
du XXe siècle. Comme le remarque Judith Butler dans Le récit de soi, « la façon dont nous sommes dès l'origine interrompus par l'altérité peut nous rendre incapables de donner une clôture narrative à nos vies » et dans cette perspective, c'est justement « le fantasme d'une maîtrise impossible » qu'il faut craindre (Butler, 2007 : 65-66). Une fois éliminée l'intrigue comme source de cohérence et moyen de maîtrise, restent les attentes et les contraintes inhérentes aux genres littéraires qui seraient susceptibles de menacer la quête de la vérité du sujet en proposant de celui-ci une conception figée. Dès lors, l'hybridité générique devient aussi le gage d'une recherche authentique de la vérité spirituelle.

Dans le dernier chapitre, ce qui prend forme et se transmet entre les femmes réunies - et qui reste difficile à mettre par écrit - se manifeste surtout grâce au dispositif des témoignages partagés et entremêlés. L'enquête a ainsi subi une nouvelle transformation - enquête historique, enquête du passe familial et individuel, elle porte fruit finalement lorsqu'elle devient enquête de soi, tout en dépassant l'introspection et l'auto-analyse par la dimension collective du partage. Pour Judith Butler, l'acte de narration présuppose un Autre, et la capacité à l'autoréférence émerge dans la relation à l'autre (Butler, 2007 : 82-83). Alors que Butler insiste sur l'interpellation, Belinda Cannone, dans la conclusion de son ouvrage Narrations de la vie intérieure, propose comme modèle, en citant les propos de Saramago, une voix d'auteur qui ne serait pas «égotiste» mais "polyphonique»; «ce que portent ces voix multiples qui se croisent en lui est un savoir commun», affirme-t-elle (Cannone, 2001 : 106). Dans Le complexe de la sorcière, la place de cet autre dont la présence s'avère essentielle est d'abord tenue par l'inquisiteur historique, par ses avatars modernes (plutôt dispositifs que personnes) ou par l'inquisiteur intériorisé, ce doute éternel sur sa propre légitimité qui ronge la psyché des femmes. Grâce à l'écriture conçue comme un travail sur soi, comme le cadre d'une transformation affectant l'intériorité, on arrive à la libération d'une force psychique qui était auparavant entravée. Cette libération est facilitée, ou peut-être même rendue possible, par l'existence des relations à l'autre qui dénoncent la logique de l'inquisition - l'autre peut être un(e) ami(e), un(e) partenaire de vie ou de dialogue, mais aussi une part de soi à laquelle on va rendre hommage, pour la rendre visible et lui donner une place légitime. 


\section{CONCLUSION : VERS UNE NOUVELLE ÉCRITURE DE L'INTÉRIORITÉ ?}

Comme nous avons pu constater à travers notre analyse, dans Le complexe de la sorcière l'écriture est le moyen par lequel on arrive à une transformation intérieure, et cette écriture est conçue comme une enquête sur la confrontation de l'inquisiteur et de la sorcière. Mais le mot inquisition signifiait justement "enquête, recherche», et l'enquête, comme le rappelle Laurent Demanze, est en effet basée sur le «paradigme inquisitorial» (Demanze, 2019 : 27). Un changement a pourtant eu lieu, dont hérite le livre d'Isabelle Sorente : si au XIX ${ }^{\text {e }}$ siècle ce paradigme est en relation avec « une ambition politique de normalisation du corps social», les écrivains contemporains pratiquent l'enquête d'une manière différente : «Il s'agit pour eux d'emprunter au paradigme inquisitorial, mais pour interroger les violences politiques, traquer les blessures historiques et mettre en évidence les tensions sociales. Émerge là une politique de la littérature » (Demanze, 2019 : 27).

Comment alors penser l'articulation entre l'enquête comme modèle d'écriture, et la transformation intérieure dont le texte témoigne ? En affirmant que le texte littéraire est le lieu de la construction de soi, nous ne faisons que redire une vérité acquise, qui retrouve sa formulation la plus célèbre dans les ouvrages de Paul Ricœur, sous forme de «l'identité narrative » ainsi que de la «triple mimèsis ». Rappelons brièvement que dans Temps et récit Ricœur distingue les trois moments de la mimèsis en définissant la mimèsis II par sa fonction de médiation entre les deux autres, et se propose de « montrer le rôle médiateur de ce temps de la mise en intrigue entre les aspects temporels préfigurés dans le champ pratique et la refiguration de notre expérience temporelle par ce temps construit » (Ricœur, 1983 : 107). Dans un texte tel que Le complexe de la sorcière, le problème est justement posé par la nature indécise de l'acte de configuration, ainsi que par le statut qu'il faudrait accorder à l'instance auctoriale.

Dans Soi-même comme un autre, en abordant la problématique du rapport entre auteur, narrateur et personnage, Ricœur affirme que l'équivocité de la notion d'auteur doit être "préservée plutôt que résolue », précisant que, d'une part, « [e]n faisant le récit d'une vie dont je ne suis pas l'auteur quant à l'existence, je m'en fais le coauteur quant au sens », alors que d'autre part, « en sens inverse, maints philosophes stoïciens ont interprété la vie elle-même, la vie vécue, comme la tenue d'un rôle dans une pièce que nous n'avons pas écrite et dont l'auteur, par conséquent, recule au-delà du rôle » (Ricœur, 1990 : 191). 
Le refus de l'intrigue, qui paraît court-circuiter la mimèsis II dans les « romans documentaires » contemporains, tout en faisant paradoxalement consolider le pouvoir de configuration textuelle, œuvre dans le sens de convergence des deux stades d'expérience pratique (mimèsis I et III), appelée par cette nouvelle politique de la littérature. Là où la violence subie ôte le pouvoir de la personne sur sa propre vie, la configuration textuelle lui permet d'en redevenir l'auteur quant à son sens. Le modèle adopté par Isabelle Sorente, mais aussi par d'autres romancières contemporaines qui choisissent de (re)configurer leur identité par l'écriture, de reprendre possession de leur propre histoire en l'écrivant (ce qui est important surtout lorsqu'il est question d'expériences traumatiques et/ou initiatiques) peut se présenter sous la forme d'un énoncé performatif : "Je suis moi-même lectrice et interprète de mon passé, et par suite auteure de ma vie».

\section{Nađa Đurić}

LITERARY INVESTIGATION, IN SEARCH OF ONESELF: ISABELLE SORENTE, THE WITCH COMPLEX

Summary

In The Witch Complex (2020), Isabelle Sorente tells how the image of the witch bursts into her life and into her writing, and leads her to question the psychic impact of witch hunts, as well as its tacit transmission to later generations. If contemporary literature "readily lays claim to inquiry as a writing process" (Laurent Demanze, Un nouvel âge de l'enquête), The Witch Complex illustrates this trend by combining historical inquiry, exploration of family memory, self-analysis and introspection. In our analysis of Isabelle Sorente's latest novel, we will turn our attention to this particular mode of literary representation of the inner life, where writing conceived as an investigation becomes the vehicle of an inner transformation. By pursuing a parallel analysis of the author's growing awareness of the witch hunts and of her own subjugation to the witch complex, and of the generic transformations that the text undergoes, we will see that the investigation first transforms the novel into an essay, and brings it closer to the notion of literature applied to psychoanalysis, as defined by P. Bayard. When the story unexpectedly turns to the author's adolescence and the traumatic experiences that marked her but have since been forgotten, we will see how the text further transforms when recalling the personal and family past, instead of being the culmination of storytelling, opens up the text to the stories of others, thus confirming the thesis of Judith Butler on the importance of the other for the act of storytelling. Ending on a series of reflections inspired by the various ways in which one can regain 
one's feminine truth, the text refuses any traditional narrative closure in its pursuit of an authentic search for spiritual truth, and its generic hybridity can be seen as serving the same purpose. Finally, the reflections of P. Ricœur will allow us to articulate the relationship between documentary literature and the construction of the subject. Where the violence suffered takes away the power of the person over her own life, the textual configuration allows her to once again become the author of its meaning.

Key words: Isabelle Sorente, Le complexe de la sorcière (The Witch Complex), investigation, women, generic hybridity, witches, 21st century French literature, inner life.

\section{RÉFÉRENCES BIBLIOGRAPHIQUES}

Bayard, P. (2012). Peut-on appliquer la littérature à la psychanalyse ? Paris : Les Éditions de minuit.

Butler, J. (2007). Le récit de soi. Traduit de l'anglais par Bruno Ambroise et Valérie Aucoutourier. Paris : Presses Universitaires de France.

Cannone, B. (2001). Narrations de la vie intérieure. Paris: Presses Universitaires de France.

Chollet, M. (2018). Sorcières : la puissance invaincue des femmes. Paris : Zones.

Demanze, L. (2019). Un nouvel âge de l'enquête : portraits de l'écrivain contemporain en enquêteur. Paris : Éditions Corti.

Eliade, M. (1978). Quelques observations sur la sorcellerie européenne. In: Occultisme, sorcellerie et modes culturelles. Traduit de l'anglais par Jean Malaquais. Paris : Gallimard. 93-124.

Jung, C. G. (1987). L'homme à la découverte de son âme: structure et fonctionnement de l'inconscient. Préfaces et adaptation par le $\mathrm{D}^{\mathrm{r}}$ Roland Cahen. Paris : Albin Michel.

Les Sorcières : Histoire d'une renaissance (no 4, novembre-décembre 2019). Le Point Référénces, Hors série.

Michelet, J. (1878). La sorcière. Paris : Calmann Lévy.

Ricœur, P. (1983). Temps et récit 1 : L'intrigue et le récit historique. Paris: Éditions du Seuil.

Ricœur, P. (1990). Soi-même comme un autre. Paris : Éditions du Seuil.

Sorente, I. (2015). La faille. Paris : JC Lattès.

Sorente, I. (2020). Le complexe de la sorcière. Paris : JC Lattès. 
